\title{
Alternative Alphas from Hedge Fund ETF Speculation
}

\author{
Peter C. L. Lin 1,2 \\ ${ }^{1}$ Financial Engineering Division, School of Systems and Enterprises, Stevens Institute of Technology, Hoboken, \\ USA \\ ${ }^{2}$ Gamma Paradigm Capital, New York, USA \\ Email: peter.lin@stevens.edu
}

Received 16 September 2015; accepted 14 February 2016; published 17 February 2016

Copyright (c) 2016 by author and Scientific Research Publishing Inc.

This work is licensed under the Creative Commons Attribution International License (CC BY).

http://creativecommons.org/licenses/by/4.0/

(c) (i) Open Access

\begin{abstract}
Alternative alpha represents risk-adjust absolute return of an alternative investing instrument regressed on alternative risk factors. Over the years, the definition is extended to the absolute return generated from alternative asset speculation-long-only or long-short strategy on alternative assets to generate additional return on top of existence alpha. In this article, we examine and propose a model with state-dependent stochastic differential equations based on Gaussian mixture model and multi-class Gaussian-kernel support vector machine to analyze hedge fund ETF alpha. We provide a new type of long-short speculation which trades on hedge fund strategies. This long-short alternative portfolio is build based on a Sharpe-ratio-like alpha ratio optimization program, and the historical performance from the portfolio shows statistically significant improvement adding to existing alphas. For passive investors, the portfolio also yields a simple portable alpha strategy which outperforms the S\&P 500 benchmark return by $7.8 \%$ since 2012 .
\end{abstract}

\section{Keywords}

Alternative ETFs, Index Investing, Hedge Fund Strategies, Quantitative Portfolio Management

\section{Introduction}

On March 25, 2009, Index IQ issued a hedge multi-strategy tracker exchange-traded fund (ETF) (ticker: QAI) to replicate the risk-adjusted returns of hedge funds covering various strategies including long-short equities, global macro, market neutral, event-driven, fixed-income arbitrage, and emerging markets. With a structure of fund of primary assets, QAI aims to statistically track the characteristics of IQ Hedge Multi-Strategy Index which is a multi-strategy hedge fund index generated by publicly available hedge fund performance. In the same year, Index IQ launched hedge fund replication products covering specific strategies: a macro tracker ETF 
(ticker: MCRO), a merger arbitrage ETF (ticker: MNA), and a real return ETF (ticker: CPI). Compared to hedge funds or hedge fund-style mutual funds, ETFs provide lower cost and higher liquidity for investors and the benefit for short selling.

The question is: can a standardized exchange-traded fund with pre-defined trading model generates stable and positive alpha comparing to a hedge fund or a fund of hedge funds? A bigger challenge, which is the key focus for this article, is whether speculating on hedge fund ETFs can generate alternative alphas additional riskadjusted absolute returns on top of existing alphas. In Fung and Hsieh [1], a risk-factor based hedge fund research provides a guideline to analyze and compare hedge fund strategies, and an alternative-alpha concept was first proposed to distinguish outperformed hedge funds to others. As the concept was used for a passive performance measurement between hedge funds, it can also be utilized as an active investing goal under a fund of hedge funds strategy paradigm. For example, Blackstone [2] in 2012 set up a fund of hedge funds to pursue alternative alpha through diversified equity hedge fund strategies. However, this long-only alternative alpha generating model still shows high market beta ${ }^{1}$. Hence, with the ability to short sale ETFs, we aim to provide a long-short alternative alpha portfolio which is designed to short sell bearish hedge fund strategies. This longshort hedge fund ETF portfolio will provide an opportunity to speculate on investing strategies, not just on assets.

Fung and Hsieh [3] analyzed the alternative alphas from a long-short equity strategy. By utilizing the Hedge Fund Research Monthly Indexes (HFRI) Equity Hedge Index, Lipper TASS Database Weighted Index, and MSCI Long Bias Index, the authors examined the ex post distribution of alternative alphas. After building the Fama-French model ${ }^{2}$, the excess kurtosis of the alphas reduces significantly, and the alternative alpha dynamics can be captured by a AR(1)-GARCH(1,1) model. Furthermore, the two-factor Fama-French model ${ }^{3}$ on these indexes has R-square of at least $81 \%$, which indicates that the equity long-short strategy can be captured adequately by this model for two reasons: the small cap minus big cap (SMB) factor is essentially an equity long-short strategy, and the market portfolio is assumed to be an equity portfolio. Consequently, this particular hedge fund strategy might be replicated statistically. Despite the promising results for quantifying alternative alphas and the possibility of applying these results to portfolio diversification, these hedge fund indexes and the Fame-French model merely provide an indirect guideline for alternative investments. The actual performance of the portfolio depends on the actual investable vehicles and the investable alternative investing strategies.

With the focus on hedge fund ETFs, we first examine the possibility of modeling hedge fund ETF alphas. We show that alpha distributions have multiple peak and can be fitted with Gaussian mixture models [4]. Labeled data are then mapped back to the time series data and show the state-dependent characteristic. As state dependent dynamics with classified labels has no closed-form solution and the computation of mean and variance would be conditioned on a sequence of stopping times, we propose a state-dependent dynamics consist of a mean-reverting Ornstein-Uhlenbeck process and support vector machine (SVM) [4] controlled parameters. We refer this model to SVM-OU framework. The model framework shows to capture the Gaussian mixture distributions of the extracted rolling hedge fund ETF alphas.

Based on the alpha state-dependent model framework, a long-short hedge fund ETF portfolio is constructed based on an optimization program to maximize Sharpe-ratio-like alpha ratio. The alpha ratio computes the expected increment/decrement of future portfolio alpha and adjusts it by ex ante alpha volatility. We show that the optimum weight of each hedge fund ETF should be proportional to alpha increment and inverse proportional to the square of alpha volatility. Since the optimization dependents on the SVM-OU model simulation, we refer the portfolio constructed by the optimization program to SVM-OU long-short hedge fund ETF portfolio (SVM-OU portfolio). Followed by monthly portfolio construction, we compare the portfolio performance to another three strategies. While HFRI Fund Weighed Composite Index and an equality weighted long-only hedge fund ETF portfolio show negative alphas from Jan 2012 to Aug 2014, our SVM-OU portfolio provides positive alpha regressed on S\&P 500 Index portfolio. Furthermore, SVM-OU portfolio shows positive alternative alpha regressed on HRFI Fund Weighted Composite Index. The historical portfolio performance from 2012 to 2014 has Sharpe ratio 1.22.

With stable and positive risk-adjusted absolute returns, a passive benchmark portfolio manager might invest

\footnotetext{
${ }^{1}$ Based on the monthly data from Apr 2012 to Aug2014, the market beta is 0.36 .

${ }^{2}$ In the Fame-French model, the factors are the excess return of the market (MKT), the spread between small cap and large cap stocks (SMB) and the spread between high book-to-market and low book-to-market stocks (HML).

${ }^{3}$ Fame-French two-factor model includes only MKT and SMB factors.
} 
in the strategy after gaining more capital flexibility by switching out cash positions to futures positions. This alpha generating and transporting process is called portable alpha. The portable alpha strategy works based on the lower margin requirement for investing in futures rather than underlying assets. Fung and Hsieh [3] transport the alternative alpha from the long-short equity strategy to an S\&P 500 portfolio after replacing S\&P 500 Index position with S\&P 500 Index futures. For a more thorough analysis, Kung and Pohlman [5] detailed the portable alpha procedure and concluded that the portable alpha technique generates better performance when the alpha is generated from different asset classes with negative correlation or low beta to the targeted portfolio. Hence, considering the case of S\&P 500 Index portfolio with, for example, HFRI Fund Weighted Composite Index, the portable alpha advantage would disappear because of high correlation between S\&P 500 Index and HFRI Index. In light of the empirical observations, we show that by transporting SVM-OU alternative alpha to an equity portfolio, the portable alpha strategy is sustainable. Despite the relatively short history, the enhanced portfolio performance validates the value of alternative alphas in benchmark investing.

\section{Modeling Alpha Dynamics}

The returns of hedge fund ETFs are positively correlated with S\&P 500. In Panel A of Table 1, though the means are significantly above zero, high kurtosis indicates that return distributions are not normal. Given high return volatility, performance over the years is not stable; hence, including hedge fund ETFs into an equity portfolio naively in order to improve performance might be detrimental. However, directional long-only strategy in hedge fund products are not our biggest concern. We would like to analyze the alpha each hedge fund ETF generates to the market and quantify how each hedge fund ETF generated alpha can contribute to alternative investing. We aim to help pick hedge fund ETFs which might have better performance in the future and build an active alternative portfolio for extracting stable and positive alternative alphas.

We first examine a simple one-factor dynamic model by rolling linear regressing hedge fund ETFs on S\&P 500 Index ETF. We conduct the linear regression on daily returns for a 252-trading-day rolling window. Equation (1) represents the Hedge-Fund-on-Market alternative alpha:

$$
R_{H F}=\alpha+\beta \times R_{M K T}+\varepsilon
$$

Panel B in Table 1 shows the statistics of the rolling alphas extracted from Equation (1). Comparing to hedge fund ETF daily returns, kurtosis values are significantly reduced to the level of a normal distribution. However, further investigation in Figure 1 shows that the alpha distributions reveal multiple peaks for non-normal distribution. Hence, we propose to fit the historical alpha distributions with Gaussian mixture models. A

Table 1. Hedge fund ETFs: Daily return statistics and rolling alpha statistics. (a) 2010/01/01 to 2014/08/31 Daily Return Statistics (in Basis Points); (b) 2011/01/01 TO 2014/08/31 252-Day Rolling Alpha Statistics (in Basis Points).

(a)

\begin{tabular}{ccccc}
\hline & QAI & MNA & CPI & MCRO \\
\hline Mean & 1.3456 & 0.9933 & 0.6021 & 0.4507 \\
Standard deviation & 42.1649 & 59.8359 & 23.6789 & 46.8892 \\
Skewness & -0.7638 & -0.2748 & -0.0589 & -0.5607 \\
Kurtosis & 35.4299 & 9.7479 & 6.5084 & 13.1548 \\
Correlation & 0.5313 & 0.4230 & 0.2146 & 0.5438 \\
\hline & & (b) & & \\
\hline Mean & QAI & MNA & CPI & MCRO \\
\hline Standard deviation & 1.7765 & 1.6372 & 0.5036 & 1.0515 \\
Skewness & 0.7495 & 1.3820 & 0.6065 & 1.4254 \\
Kurtosis & 0.3531 & -0.3376 & -0.4209 & -0.0084 \\
Correlation & 3.4275 & 2.0417 & 2.3686 & 2.2310 \\
\hline
\end{tabular}




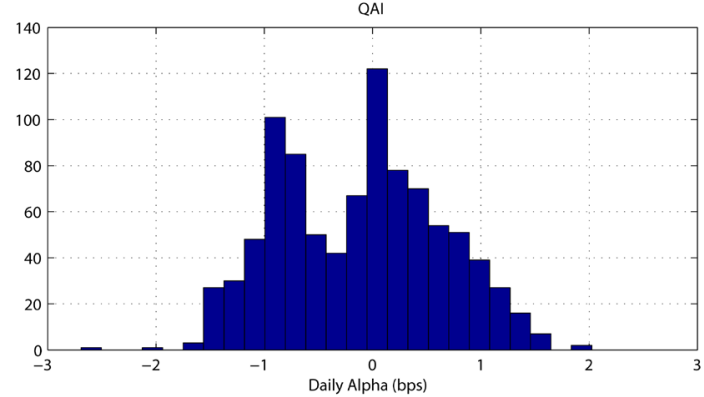

CPI

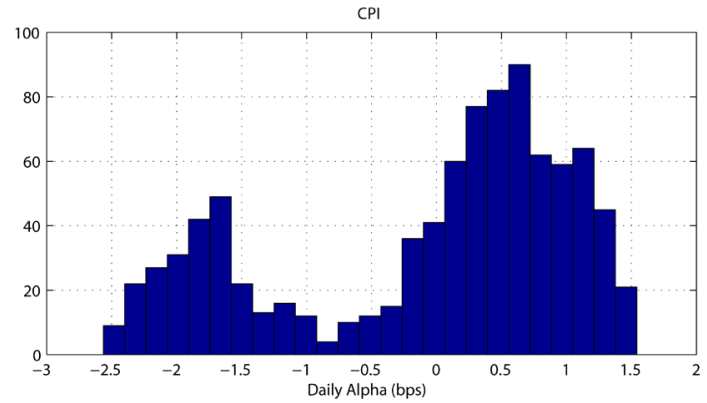

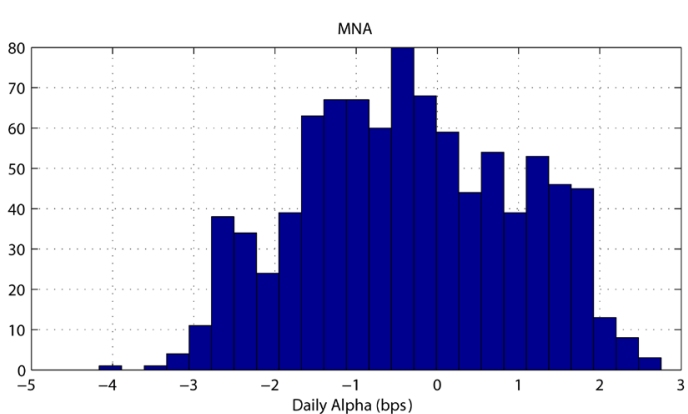

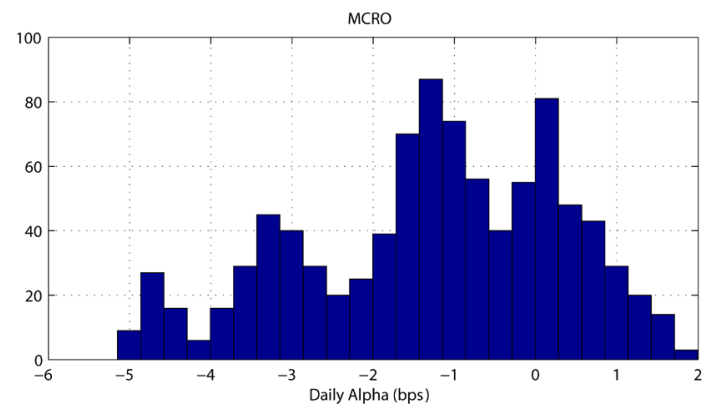

Figure 1. 252-Day Rolling Alpha Distributions from 2011/01/01 to 2014/08/31. The plots show non-Gaussian distributions for alphas with the statistics generated in Panel B of Table 1.

Gaussian mixture model with probability density function is shown in Equation (2):

$$
p(\theta)=\sum_{n=1}^{N} \theta_{n} f\left(\mu_{n}, v_{n}^{2}\right)
$$

where $f$ is the density function of a normal random variable with mean $\mu_{n}$ and variance $v_{n}^{2}$, and

$$
\sum_{n=1}^{N} \theta_{n}=1
$$

Please refer to Bishop [4] for more details about Gaussian mixture models.

With a fixed number $N$, the parameters for each Gaussian component can be determined by expectationmaximization algorithm. If $N$ is unknown for the case of an unsupervised classification case, the number of Gaussian components needs be determined by cross-model evaluation. There are two common methods for model selection. Akaike Information Criterion (AIC) [6] measures the relative quality of a model by evaluating the information loss with a pre-defined penalty term on the number of components. Similarly, Bayesian Information Criterion (BIC) [7] imposes higher penalty on a finite selection of exponential family models. Calculating AIC/BIC helps to identify the proper number of components in each Gaussian mixture distribution which then be applied to identify sample classes. The result yields that QAI has 2 components, MNA has 3 components, CPI has 3 components, and MCRO has 4 components for the data from the beginning of 2011 to the August 2014.

We can now label each component from a fitted Gaussian mixture distribution to the alpha time series. Figure 2 presents the clusters in different colors and symbols. Horizontal clusters can be easily identified, which suggests that hedge fund ETF alpha dynamics follow state-dependent Markov processes. As finite numbers of component are determined by Gaussian mixture models, the coefficients of a state-dependent process will be chosen from a finite set which are controlled by a supervised multi-class Support Vector Machine (SVM).

For a Gaussian mixture model with only two components, a two-class SVM suffices to choose the boundary based on the kernel function which maximizes the margin between the two classes. Gaussian kernel is adapted in our two-class SVM to incorporate the cases that the classes cannot be separated by a linear function, for example, one class might be surrounded by the other class. With $N>2$ multi-class case, the most commonly used approach was proposed by Vapnik in [8] based on $N$ two-class SVMs, and the data label is assigned to the 

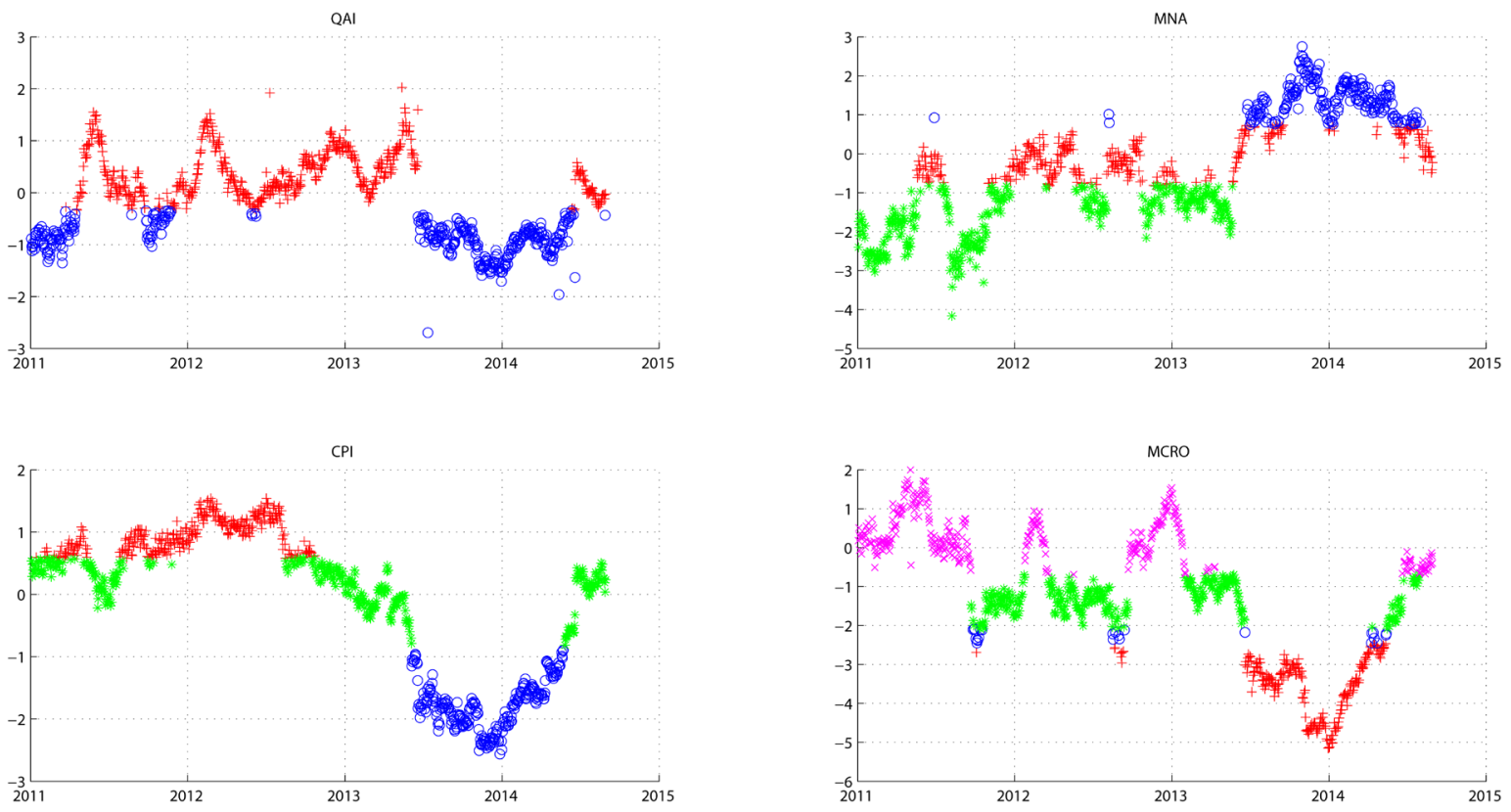

Figure 2. Labeled rolling alpha time series from 2011/01/01 to 2014/08/31.

class with highest score. The fact that the simple one-versus-the-rest approach works for our study is because each class is on the same scale and no symmetry is required in the classified data. We shall also see in the following that we can embed the calibrated multi-class SVM model into a continuous-time stochastic process.

After identifying the labeled alpha time series from Gaussian mixture models, the patterns further reveal mean-reverting characteristic. If we want to build a mean-reverting stochastic differential equation with mixed Gaussian distribution characteristic, a state-dependent Ornstein-Uhlenbeck process is an ideal candidate. With labeled states determined from a multi-class Gaussian-kernel SVM, we can identify the regimes of OrnsteinUhlenbeck processes and their coefficients. The alternative alpha dynamics $\alpha(t)$ is provided as follow:

$$
\mathrm{d} \alpha(t)=\kappa_{j}(\alpha(t))\left[\mu_{j}(\alpha(t))-\alpha(t)\right] \mathrm{d} t+\sigma_{j}(\alpha(t)) \mathrm{d} W(t)
$$

where $W(t)$ is a standard Brownian motion with

$$
j=\underset{n=1, \cdots, N}{\operatorname{argmax}} y_{n}(x)
$$

and

$$
y_{n}(x)=e_{n}\left(x, w_{n}\right)+b_{n}
$$

where $N$ is the number of Gaussian mixtures labels, and $y_{n}$ is one-versus-the-rest SVM with $e_{n}$ Gaussian kernels associated with a hyperplane defined by the normal vector $w_{n}$ and constant $b_{n}$. Then, the coefficient set for each Ornstein-Uhlenbeck process will be calibrated separately for each in-sample labeled data. Take MCRO for example, we have $N=4$, so the Ornstein-Uhlenbeck coefficient set is $\left\{\left(\kappa_{1}, \mu_{1}, \sigma_{1}\right), \cdots,\left(\kappa_{4}, \mu_{4}, \sigma_{4}\right)\right\}$, and $j$ is determined by the multi-class Gaussian-kernel SVM based on the rolling alpha at time $t$, which is $\alpha(t)$. In the following sections, we will refer this setting to SVM-OU model.

\section{Alternative Alpha}

For an active alternative portfolio management, alternative alpha represents the absolute return from speculating alternative instruments. Unlike a typical fund of hedge funds, we can short sale hedge fund with hedge fund ETF; hence, our goal is to construct an alpha long-short strategy under SVM-OU framework and compare it to a HFRI Fund Weighted Composite Index and a simple equally weighted hedge fund ETF portfolio which are both alpha long-only.

Since we expect to construct an alternative portfolio to generate stable alternative alpha, we propose a long- 
short hedge fund ETF portfolio to maximize the Sharpe-ratio-like alpha ratio:

$$
\frac{\mathbf{E}\left[\sum_{k=1}^{K} \omega_{k}\left(\alpha_{k}(T)-\alpha_{k}(0)\right)\right]}{\operatorname{Var}\left[\sum_{k=1}^{K} \omega_{k} \alpha_{k}(T)\right]}
$$

where $\alpha$ is the hedge fund ETF alpha process under SVM-OU model and $T>0$ denotes a fixed future time.

Maximizing the alpha ratio is equivalent to solving a constrained optimization problem that

$$
\max _{\pi=\left\langle\pi_{1}, \cdots, \pi_{K}\right\rangle} \sum_{k=1}^{K} \pi_{k}\left(\mathbf{E}\left[\alpha_{k}(T)\right]-\alpha_{k}(0)\right)
$$

subject to the volatility constraint

$$
\Theta=\sum_{k=1}^{K} \pi_{k}^{2} \operatorname{Var}\left[\alpha_{k}(T)\right]
$$

Let $\mu_{k}=\mathbf{E}\left[\alpha_{k}(T)\right]$ and $\sigma_{k}^{2}=\operatorname{Var}\left[\alpha_{k}(T)\right]$, and the optimization problem can be solved by the Langrangian:

$$
\max _{\pi} \sum_{k=1}^{K} \pi_{k}\left(\mu_{k}-\alpha_{k}\right)-\lambda \sum_{k=1}^{K} \pi_{k}^{2} \sigma_{k}^{2} .
$$

The first-order conditions for optimality yield

$$
\pi_{k}=\frac{\mu_{k}}{2 \lambda \sigma_{k}^{2}}, \quad k=1, \cdots, K
$$

where $\lambda$ serves as the leverage multiplier. Then, we construct the portfolio with weights $\omega=\left\langle\omega_{1}, \cdots, \omega_{K}\right\rangle$ and with a selected leverage multiplier $\lambda$ such that

and maintain a portfolio with no leverage that

$$
\omega_{k}=\frac{\pi_{k}}{\sum_{k=1}^{K} \pi_{k}}
$$

$$
1=\sum_{k=1}^{K}\left|\omega_{k}\right|
$$

To construct the described portfolio, we simulate under SVM-OU model for each hedge fund ETF to derive the mean and variance for a future time $T$. The optimization is under a typical mean-variance paradigm, but it conveys three important messages. First, instead of maximizing return which might be highly correlated to market return, we maximize portfolio alpha and expect a state-dependent momentum on future alpha to generate absolute return. Second, a Sharpe-ratio-like alpha ratio is adjusted by the alpha volatility. Lower alpha volatility shows better hedge fund manager's skill and better hedge fund strategy. Hence, this portfolio is built to capture a steady alpha under various market conditions. Third, we look for a long-short alternative portfolio to short sale alpha if needed. An alternative alpha therefore represents both the absolute return generated from a portfolio of alternative instruments and the absolute alpha by long-short traditional alphas. Since the optimization dependents on the SVM-OU model simulation, we refer the portfolio constructed by the optimization program to SVM-OU long-short hedge fund ETF portfolio (SVM-OU portfolio).

\section{Alternative Alpha Portfolio}

SVM-OU portfolio speculates on future alphas of hedge fund ETFs and creates long-short positions. By running a rolling window of 252 days, we calibrate the parameters for the SVM-OU processes and buy and hold the portfolio for a month. The calibration process includes adjusting the Gaussian mixture model for data labeling, SVM model training for supervised classification, and coefficients for Ornstein-Uhlenbeck processes. Using the alpha data generated starting from 2011, we compare the monthly performance of our SVM-OU alternative alpha portfolio to two other portfolios: 1) HFRI Fund Weighted Composite Index, and 2) a simple averaging 
portfolio by assigning equally positive weight to each hedge fund ETF.

The results are shown in Table 2. From 2012 to 2014, both the simple long-only averaging portfolio and HFRI portfolios generate negative alphas. The high market betas and negative alphas of these two portfolios necessitate the long-short speculation to generate sustainable alternative alpha. On the other hand, our long-short strategy based on SVM-OU model generates stable positive alphas regressed on both S\&P 500 Index and HFRI Fund Weighted Composite Index, and the low betas are both low statistically significant at $99 \%$ level. Also, the SVM-OU portfolio has better performance comparing to the simple momentum with $23 \%$ improvement on portfolio Sharpe ratio.

Despite the short history, the result shows the possibility of alternative alpha by speculating on alternative instruments. As more hedge-fund-like products become available to general public, we can expect a further decline on hedge fund long-only alphas. However, based on our result, a long-short strategy on hedge-fund strategies may have proven to be a good alternative investing strategy. With the increasing popularity of hedgefund replicate products in mutual funds, ETFs, and ETNs ${ }^{4}$, we can expect a trend of speculation on this asset class which sells various investing strategies, and the new alternative alphas might eventually replace existing alphas.

\section{Portable Alphas}

A portable alpha strategy is not new to portfolio managers. The idea is to replace cash positions with futures contracts, which only needs a lower capital requirement if trading with margin. Subsequently, portfolio managers can invest the additional capital in alpha-generating assets or strategies. As a cash position can be tracked by a futures position (for instance, S\&P 500 ETF SPY tracked by S\&P 500 mini futures) for a preset time horizon, the portable alpha strategy is expected to steam roll out additional returns while maintaining a futures portfolio with the same asset weights as the cash portfolio at the end of the time horizon. However, the implementation relies on the identification an effective alpha-generating strategy. The insight provided by Kung and Pohlman [5] indicates that the combination of low correlated assets or markets to the original cash portfolio provides better selections of alpha-generating strategies.

Though hedge funds or funds of funds are the typical choice for generating alphas, they are not suitable for a portable alpha implementation because of their long redemption periods which are too illiquid for a short-term cash-futures interchange. Hedge fund ETFs can therefore serve a better purpose without incurring similar liquidity risk. In previous sections, we show that alternative alphas generated by hedge fund ETF speculation can provide stable additional returns on S\&P 500 Index portfolio and on HFRI Fund Weighted Composite Index comparing to a long-only fund of funds multi-strategy. Combining the result of low correlation between SVMOU and the market portfolio, SVM-OU portfolio will then serve as a good alpha generating component of a portable alpha implementation.

We look at the daily returns of a portable alpha strategy by incorporating our long-short hedge fund ETF

Table 2. Portfolio alphas and betas. ( ${ }^{*}$ ) at 99\% Confidence level. (a) Alpha-regression on S\&P 500 index. (b) Alternative alpha-regression on HFRI fund weighted composite index.

(a)

\begin{tabular}{cccc}
\hline & HFR Index & Long-Only & SVM-OU \\
\hline$\alpha$ & -0.0185 & -0.1483 & 0.0267 \\
$\beta$ & $0.3631^{*}$ & $0.2478^{*}$ & $0.1141^{*}$ \\
\hline
\end{tabular}

(b)

\begin{tabular}{ccc}
\hline & Long-Only & SVM-OU \\
\hline$\alpha$ & -0.1261 & 0.0738 \\
$\beta$ & $0.6650^{*}$ & $0.2438^{*}$ \\
R-Squared & 0.679 & 0.207 \\
Sharpe Ratio & 0.99 & 1.22 \\
\hline
\end{tabular}

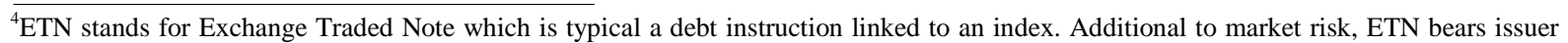
credit risk. The maturity of ETN also sets a fixed time horizon for long-term investing strategies. 


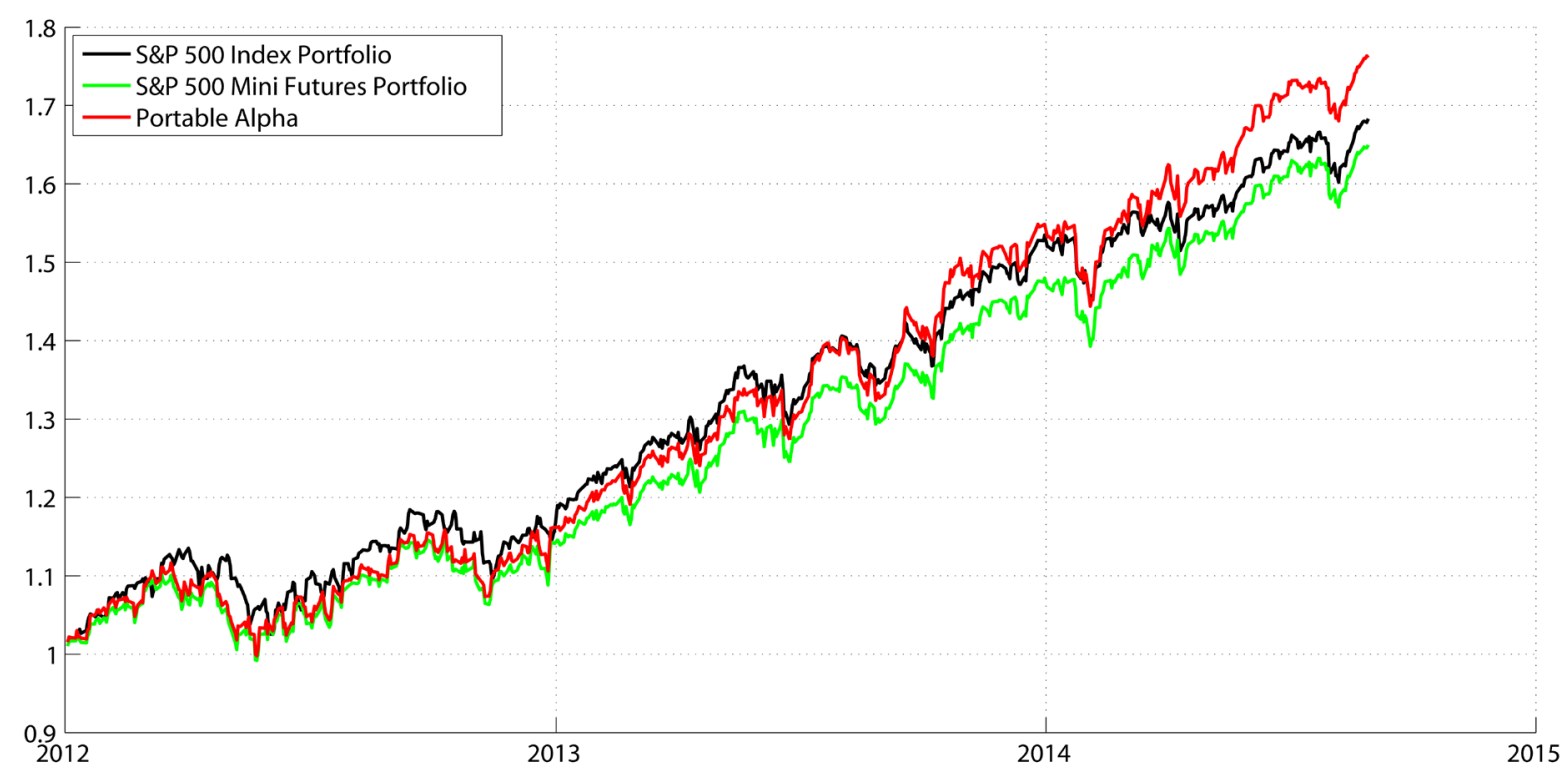

Figure 3. 2012 to 2014 Portfolio performance. total returns: 68.32\% (S\&P 500 Index), 64.97\% (S\&P 500 Futures), 76.12\% (Portable alpha portfolio).

portfolio to S\&P 500 Index portfolio. Figure 3 presents a comparison of daily S\&P 500 returns and the portable alpha implementation with S\&P mini futures combining the long-short hedge fund ETF portfolio. Back-test result shows that the SVM-OU portable alpha strategy generates additional 7.8\% return from January 2012 to August 2014 comparing to S\&P 500 Index portfolio. Benefiting from stable and positive alternative alphas with low correlation with the market, we can enhance market portfolio performance.

\section{Conclusion}

This paper introduces four hedge fund ETFs and the alternative alphas generated by speculating on hedge fund ETF alphas. Data justification starts by fitting historical rolling alpha distributions to Gaussian mixture models, and then we impose a supervised multi-class Gaussian-kernel support vector machine (SVM) classification and integrate it into state-dependent Ornstein-Uhlenbeck processes. This new framework is referred as SVM-OU model. Then, a long-short hedge fund ETF portfolio is constructed to maximize Sharpe-ratio-like alpha ratio based on the simulated ex ante alpha mean and volatility under SVM-OU model framework. From Jan 2011 to Aug 2014, the SVM-OU portfolio return shows low beta and sustainable positive alpha while HFRI Fund Weighted Composite Index and a simple equally-weighted long-only hedge fund ETF portfolio show negative alpha with much higher beta. This article provides an additional use case by implementing the SVM-OU alternative alpha in a portable alpha strategy. Comparing to S\&P 500 Index portfolio, the portable alpha strategy generates additional 7.8\% return from January 2012 to August 2014. A generalization of this long-short alpha speculation to other alternative assets is planned for future investigation.

\section{References}

[1] Akaike, H. (1974) A New Look at the Statistical Model Identification. IEEE Transactions on Automatic Control, 19, 716-723. http://dx.doi.org/10.1109/TAC.1974.1100705

[2] Bishop, C.M. (2007) Pattern Recognition and Machine Learning. Springer.

[3] Blackstone (2014) Blackstone Alternative Alpha Funds. blackstone.com

[4] Fung, W. and Hsieh, D.A. (2004) Extracting Portable Alphas from Equity Long-Short Hedge Funds. Journal of Investment Management, 2, 57-75.

[5] Fung, W. and Hsieh, D.A. (2003) The Risks in Hedge Fund Strategies: Alternative Alphas and Alternative Betas. Euromoney Institutional Investors PLC, 72-87.

[6] Kung, E. and Pohlman, L. (2004) Portable Alpha: Philosophy, Process \& Performance. Journal of Portfolio Manage- 
ment, 30, 78-87. http://dx.doi.org/10.3905/jpm.2004.412322

[7] Schwarz, G. (1978) Estimating the Dimension of a Model. The Annals of Statistics, 6, 461-464. http://dx.doi.org/10.1214/aos/1176344136

[8] Vapnik, V.N. (1998) Statistical Learning Theory. Wiley. 